\title{
Bestimmung des Körpergewichtes mit Hilfe des Brustumfanges beim Fleckviehjungrind
}

\author{
Herrn Prof. Drs. h. c. Franz Pirchner PhD, zum 75. Geburtstag gewidmet
}

\section{Summary}

Title of the paper: Prediction of the body weight of Simmental heifers using heart girth measurements The decision of the date of the first insemination of heifers is mainly based on the weight. In most of the dairy farm no scale exists, therefore a trial had been started to estimate a regression equation for the prediction of the body weight by the heart girth measurement.

On seven farms the weight, heart girth and hip height of 324 heifers had been collected. The age of the heifers had been between 8 and 26 months. Additionally the results of heart girth and weight of 347 heifers out of 8 farms had been included.

The analysis of the farm influence, adjusted by age, showed high significant effects in hip height, heart girth and weight. Heifers, grown up on farms with higher milk yield, had a larger size.

For predicting the body weight of Simmental heifers using the heart girth measurement (BU) the following equation had been analyzed: $\hat{Y}_{G E W}=137.6-2.647 * X_{B U}+0.024 * X_{B U}{ }^{2}$. The $\mathrm{R}^{2}$-value is 0.94 .

Key Words: prediction of body weight, heifer, Simmental, heart girth, farm effect

\section{Zusammenfassung}

Das Gewicht von Jungrindern wird vor allem bei der Entscheidung des Besamungszeitpunktes benötigt. Da in vielen Milchviehbetrieben keine Waage vorhanden ist, wurden Messungen des Brustumfanges, der Kreuzbeinhöhe sowie Wägungen von Jungrindern vorgenommen, um eine Regressionsgleichung zur Gewichtsschätzung zu berechnen.

In sieben Betrieben wurden an 324 Jungrindern das Gewicht, der Brustumfang und die Kreuzbeinhöhe erfasst. Die Tiere waren zwischen 8 und 26 Monate alt. Weiterhin wurden die Ergebnisse von 347 Jungrindern aus 8 Betrieben in die Auswertung mit einbezogen, von denen nur das Gewicht und die Brustumfangsmesswerte vorlagen.

Die Analyse des Betriebseinflusses unter Berücksichtigung des Alters erbrachte hochsignifikante Differenzen für die Merkmale Gewicht, Brustumfang und Kreuzbeinhöhe zwischen den Betrieben. Betriebe mit höherer Milchleistung haben deutlich höhere Werte in den Merkmalen Kreuzbeinhöhe, Brustumfang und Gewicht.

Zur Vorausschätzung des Gewichtes mit Hilfe des Brustumfangs wurde für Fleckviehjungrinder folgende Gleichung geschätzt: $\hat{Y}_{G E W}=137,6-2,647 * X_{B U}+0,024 * X_{B U}^{2}$

Schlüsselwörter: Gewichtsschätzung, Jungrind, Fleckvieh, Brustumfang, Betriebseinfluss

1. $\quad$ Einleitung

Nach SWANSON (1960) ist die optimale Wachstumskurve bei der Aufzucht von Jungrindern abhängig vom Erreichen des optimalen Gewichts beim gewünschten Abkalbealter zur Ausschöpfung des genetischen Potentials in der ersten Laktation und minimaler Kosten. 
Das Gewicht beim Erstabkalben steht in direktem Zusammenhang mit dem Belegungsgewicht, das zwischen 62 und 65\% vom Gewicht bei der Erstabkalbung (HEINRICHS et al., 1998; HOFFMANN et al., 1996; PLATEN und KROCKER, 1998; VEAUTHIER und ACHLER, 1999) betragen soll.

Diese und andere Gründe machen die Körpergewichtsbestimmung notwendig, was am sichersten durch Wägung geschieht. Dies ist nicht immer möglich. Bereits in dem klassischen Buch der Rinderzucht von DUERST (1931) wird über Bemühungen zur Gewichtsbestimmung von Rindern mit Hilfe der Barymetrik berichtet, wobei aus erfassten einzelnen Körpermaßen und deren unterschiedlicher Verknüpfung eine Gewichtsschätzung erreicht werden sollte.

Da in vielen Betrieben keine Waagen zur Verfügung stehen gab es auch später immer wieder Untersuchungen über die Beziehungen zwischen Körpermaßen und dem Lebendgewicht (BREM, 1998).

Zur Bestimmung des Kuhgewichts in der 1. Laktation, im Rahmen der Nachkommenprüfung von Besamungsbullen der Rasse Fleckvieh, wog und maß FLATNITZER (1968) 914 Jungkühe. Die statistische Analyse seiner Daten zur Schätzung des Gewichtes $\left(\hat{Y}_{G E W}\right)$ erbrachte unter Berücksichtigung des Brustumfanges $\left(X_{B U}\right)$ folgende Regressionsgleichung: $\hat{Y}_{G E W}=5,71 * X_{B U}-556$.

Weitere erfasste Körpermaße wurden nicht berücksichtigt, da diese nicht zu einer signifikanten Erhöhung der Genauigkeit der Vorhersage des Gewichtes führten.

LIPPERT et al. (1994) werteten Messungen an Fleckviehkühen in der ersten Laktation aus. Sie konnten feststellen, dass für die Gewichtsschätzung nicht nur der Brustumfang, sondern auch der Keulenumfang von Bedeutung ist.

Nach BREM (1998) wird bei Fleckviehjungkühen der Brustumfang für eine Schätzgleichung wie folgt verwendet: $\hat{Y}_{G E W}=5,71 * X_{B U}-557$.

Für die Rasse Braunvieh berechneten ALPS und STEURER (1983) unter Berücksichtigung des Brustumfangs und der Mittelhandlänge $\left(X_{M H L}\right)$ folgende Schätzgleichung: $\hat{Y}_{G E W}=5,05 * X_{B U}+3,48 * X_{M H L}-726,5$.

In neueren Untersuchungen für die Gewichtsschätzung bei Jungrindern liegen die Ergebnisse von HEINRICHS et al. (1992) vor, die in den USA an 1600 Jungrindern der Rasse Holstein Friesian (HF) das Gewicht und die Körpermaße Brustumfang, Widerristhöhe, Kreuzbeinhöhe und Körperlänge erfassten. Unter Berücksichtigung der linearen und quadratischen Komponente des Brustumfanges wurde für die Gewichtsschätzung eine Genauigkeit von $\mathrm{R}^{2}=0,99$ erzielt.

Mit Hilfe der Regressionsgleichung: $\hat{Y}_{G E W}=102,71-2,876 * X_{B U}+0,02655 * X_{B U}{ }^{2}$ wurde von ihnen ein Bandmaß zur Gewichtsbestimmung bei Jungrindern entwickelt. In vorliegender Untersuchung soll an Jungrindern der Rasse Fleckvieh (FV) geprüft werden, in wieweit die von HEINRICHS et al. (1992) für HF berechnete Regressionsgleichung auf Fleckvieh übertragbar ist.

\section{2. $\quad$ Material und Methode}

In sieben Betrieben wurden 324 Jungrinder der Rasse Fleckvieh im Jahre 2000 von einem Team gemessen und gewogen. Im einzelnen wurden die Kreuzbeinhöhe, der Brustumfang (beide Messpunkte nach BREM (1998)), das Gewicht und das Alter pro 
Einzeltier ermittelt. Die Auswahl der Betriebe erfolgte zufällig. Voraussetzung waren jedoch mindestens 50 Kühe pro Betrieb. Zusätzlich musste gewährleistet sein, dass ein Treibgang zum Wiegen und ein Selbstfanggitter zur Fixierung der Tiere bei der Messung vorhanden waren. An Stelle der Widerristhöhe wurde die Kreuzbeinhöhe gemessen, da nach ALPS (1985) die Kreuzbeinhöhe mit einer größeren Genauigkeit als die Widerristhöhe erfasst werden kann. Zusätzlich wurden Daten von 347 Rindern aus 8 Betrieben mit in die Auswertung einbezogen, die 1999 vom Beratungsdienst Ostalb in Ellwangen erhoben wurden.

Die Datenauswertung wurde mit der PC-Version des Programmpaketes SAS (Statistic Analysis System, 1990) durchgeführt. Zur Schätzung des Betriebseinflusses wurden die Daten des selbstermittelten Teilmaterials nach folgendem Modell analysiert:

dabei bedeutet:

$$
Y_{i j}=\mu+B_{i}+\operatorname{cov}_{A}+e_{i j}
$$

$$
\begin{aligned}
& \mu=\text { Erwartungswert von } Y_{i j} \\
& B_{j}=\text { Einfluß des i-ten Betriebes }(\mathrm{i}=1, . ., 7) \\
& \operatorname{cov}_{A}=\text { Alter als Kovariable } \\
& e_{i j}=\text { Restfehler }
\end{aligned}
$$

Zur Berechnung der Regressionsgleichung des Brustumfangs auf das Gewicht wurden dieselben Daten nach folgendem Modell analysiert:

dabei bedeutet:

$$
Y_{i j}=\mu+B_{i}+\operatorname{cov}_{B U}+\operatorname{cov}_{B U}^{2}+e_{i j}
$$

$$
\begin{aligned}
& Y_{i j}=\text { Gewicht } \\
& \mu=\text { Erwartungswert des Gewichtes } Y_{i j} \\
& B_{i}=\text { Einfluß des i - ten Betriebes }(\mathrm{i}=1, . ., 14) \\
& \operatorname{cov}_{B U}=\text { Kovariable Brustumfang, linear } \\
& \operatorname{cov}_{B U}=\text { Kovariable Brustumfang, quadratisch } \\
& e_{i j}=\text { Restfehler }
\end{aligned}
$$

Da für die Entwicklung eines allgemeingültigen Maßbandes der Betriebseinfluss sehr schwierig zu berücksichtigen ist, wurde eine weitere Regressionsgleichung ohne Berücksichtigung des Betriebseinflusses am Gesamtmaterial geschätzt.

Ergebnisse und Diskussion

\section{Betriebseinfluss}

Der Betrieb hat einen hochsignifikanten Einfluss auf die Merkmale Gewicht, Brustumfang und Kreuzbeinhöhe (Tab. 1).

Nach HOFFMANN et al. (1996) wurden in Hochleistungsherden größere Kühe vorgefunden, als in Betrieben mit mittlerer Leistung. Die vorliegenden Ergebnisse bestätigen diese Aussage. So erreichten die Kühe der Herden 1, 2, und 3 mit der geringsten Kreuzbeinhöhe durchschnittliche Milchleistungen um 6500 kg Milch, wohingegen die Zuchtbetriebe mit Milchleistungen von über 8000 kg signifikant höhere Gewichte, Brustumfänge und Kreuzbeinhöhen aufweisen (Tab. 2).

Der Elitezuchtbetrieb (Nr. 6) hat ein besonderes Fütterungskonzept in der Aufzucht. Bis zum 14. Monat werden die Jungrinder intensiv und danach verhalten gefüttert. Im 
Vergleich zu den Betrieben 4 und 5 wird, wie an der Kreuzbeinhöhe erkennbar, das Höhenwachstum dieser Tiere nicht beeinflusst, jedoch das durch den Brustumfang dokumentierte Brusttiefen- und Breitenwachstum wird durch die restriktive Fütterung ab dem 14. Monat im Betrieb 6 verlangsamt.

\section{Tabelle 1}

Ergebnisse der Varianzanalyse für Gewicht, Kreuzbeinhöhe und Brustumfang in Abhängigkeit vom Betrieb (Results of the analysis of variance for body weight, hip height and heart girth depending on farm $(\mathrm{n}=324)$ )

\begin{tabular}{lrlll}
\hline Varianzursache & FG & Gewicht+) & Brustumfang+) & Kreuzbeinhöhe+) \\
\hline Betrieb & 6 & $66821^{* *}$ & $2018^{* *}$ & $816^{* *}$ \\
Kov Alter, linear & 1 & $318683^{* *}$ & $16207^{* *}$ & $4183^{* *}$ \\
Kov Alter, quadr. & 1 & $21716^{* *}$ & $4352^{* *}$ & $1619^{* *}$ \\
Rest & 315 & 1464 & 44 & 12 \\
\hline
\end{tabular}

+) Mittlere Abweichungsquadrate und Signifikanz (** $=\mathrm{p}<=0,01)$

Die Unterschiede in der Kreuzbeinhöhe werden eher durch die genetische Komponente, der Brustumfang sowohl durch die genetische als auch durch die umweltbedingte Komponente verursacht, wie aus einer Datensammlung von Heritabilitätsschätzwerten von BREM (1998) zu entnehmen ist. Auch HOFFMANN (1997) wies genetische Gründe für die Betriebsdifferenzen in der Größe der Kühe nach.

Tabelle 2

LSQ - Mittelwerte verschiedener Merkmale für die Betriebe, korrigiert auf das Alter (LSQ-Means of different traits of farms adjusted for age) $(\mathrm{n}=324)$

\begin{tabular}{ccccc}
\hline Betrieb & Milch kg* & Gewicht, kg & Brustumfang, cm & Kreuzbeinhöhe,cm \\
\hline 1 & 6420 & $438^{\mathrm{b}}$ & $180,0^{\mathrm{b}}$ & $128,9^{\mathrm{c}}$ \\
2 & 6350 & $390^{\mathrm{d}}$ & $179,0^{\mathrm{b}}$ & $128,9^{\mathrm{c}}$ \\
3 & 6670 & $419^{\mathrm{c}}$ & $178,3^{\mathrm{b}}$ & $129,4^{\mathrm{c}}$ \\
4 & 8100 & $490^{\mathrm{d}}$ & $190,0^{\mathrm{a}}$ & $133,8^{\mathrm{b}}$ \\
5 & 8250 & $502^{\mathrm{a}}$ & $191,2^{\mathrm{a}}$ & $134,6^{\mathrm{ab}}$ \\
6 & 9450 & $493^{\mathrm{a}}$ & $188,5^{\mathrm{a}}$ & $135,2^{\mathrm{a}}$ \\
7 & 7300 & $444^{\mathrm{b}}$ & $175,2^{\mathrm{c}}$ & $124,1^{\mathrm{d}}$ \\
\hline
\end{tabular}

Mit ungleichen Buchstaben gekennzeichnete LSQ-Mittelwerte sind mit einer Wahrscheinlichkeit von $\mathrm{p}<0,05$ von einander verschieden.

* Rohmittelwerte

Die Jungrinder des Betriebes 7 sind mit 124,1 Zentimeter Kreuzbeinhöhe sowohl kleiner als auch deutlich geringer im Brustumfang. Diese Daten wurden im Rahmen eines Versuches zum Vergleich verschiedener Fütterungskonzepte in der Aufzucht erhoben, was möglicherweise die Körperproportionen beeinflusst haben mag.

\section{Regressionen}

Die Regressionsberechnungen wurden mit den Daten des Gesamtmaterials von insgesamt 671 Jungrindern durchgeführt.

Die Ergebnisse der Varianzanalyse in Tabelle 3 bestätigen den Betriebseinfluss (s. Tab. 1), der an einem Teilmaterial nachgewiesen wurde.

Für die Kovariable Brustumfang konnte sowohl für die lineare, als auch die quadratische Komponente ein signifikanter Einfluss auf das Gewicht nachgewiesen werden.

Folgende Regressionsgleichungen wurden mit (FV mit BK) und ohne Berücksichtigung des Betriebseffektes (FV ohne BK) berechnet.

$\hat{Y}_{G E W}=139,4-2,876 * X_{B U}+0,0253 * X_{B U}{ }^{2} \quad$ (FV mit BK)

$\mathrm{R}^{2}=0,96$ 
$\hat{Y}_{G E W}=137,6-2,647 * X_{B U}+0,024 * X_{B U}{ }^{2}$

$\mathrm{R}^{2}=0,94$.

Tabelle 3

Ergebnisse der Varianzanalyse für das Gewicht in Abhängigkeit vom Betrieb und der Kovariablen Brustumfang (Results of the analyses of variance for body weight depending on farm and heart girth (Gesamtmaterial; $\mathrm{n}=671$ Jungrinder))

\begin{tabular}{|c|c|c|c|c|c|c|}
\hline $\begin{array}{l}\text { Varianz- } \\
\text { ursache }\end{array}$ & FG & MAQ & Sign. & FG & MAQ & Sign. \\
\hline Betrieb & 14 & 17008 & $* *$ & & & \\
\hline $\begin{array}{l}\text { Kov. Alter, } \\
\text { linear }\end{array}$ & 1 & 18756 & $* *$ & 1 & 18315 & $* *$ \\
\hline $\begin{array}{l}\text { Kov. Alter, } \\
\text { quadr. }\end{array}$ & 1 & 179833 & $* *$ & 1 & 176178 & $* *$ \\
\hline Rest & 654 & 815 & & 668 & 1155 & \\
\hline
\end{tabular}

Im Vergleich dazu führt die von HEINRICHS et al. (1992) für HF-Jungrinder berechnete Regressionskurve (Abb.) in der Jugendphase (Brustumfang bis zu 180 Zentimetern) zu niedrigeren Gewichten, um im späteren Bereich z.B. bei einem Brustumfang von 200 Zentimetern um ca. 21 Kilogramm über dem der Fleckviehkurve ohne Betriebskorrektur zu liegen.

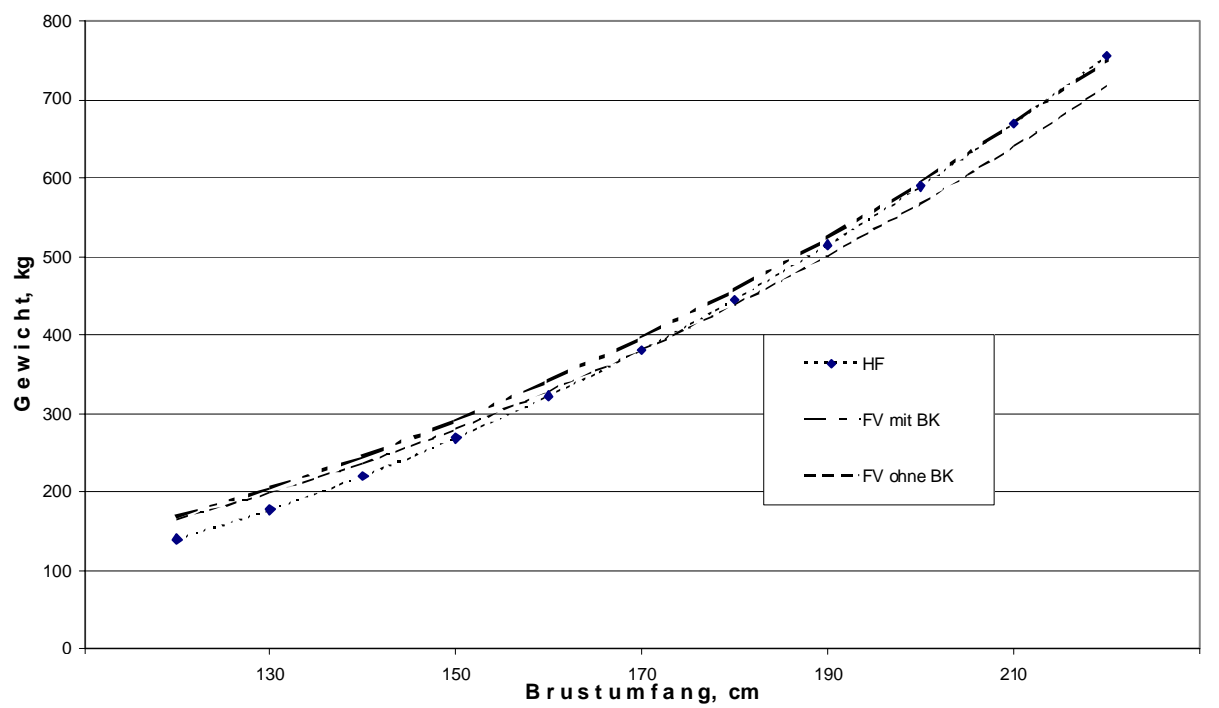

Abb.: Gewichtsschätzung in Abhängigkeit vom Brustumfang (Prediction of body weight by heart girth)

Eine Einbeziehung der Kreuzbeinhöhe in die Regressionsgleichung erbrachte keine höhere Schätzgenauigkeit.

Bei Berechnung der Regressionsgleichung wurde ein $\mathrm{R}^{2}$-Wert von 0,94 erreicht. Die Einbeziehung des Betriebes in das Modell erhöhte den $\mathrm{R}^{2}$-Wert auf 0,96. Da eine Betriebskorrektur beim praktischen Einsatz auf den Betrieben nicht möglich ist, wird für das Fleckvieh zur Schätzung des Gewichtes die Repressionsgleichung ohne Betriebskorrektur empfohlen.

Der für HF-Jungrinder angenommene Brustumfang von 200 Zentimetern entspricht einem Gewicht von 589 kg, welches das Optimalgewicht beim Erstabkalben ist, wie es aufgrund der Ergebnisse neuerer Untersuchungen von GRUMMER et al. (1995) und HOFFMANN et al. (1996) empfohlen wird. 
Adulte Fleckviehkühe haben nach WILLEKE et al. (2001) ein Gewicht von 750 kg. Da das Erstabkalbegewicht ca. 85 Prozent vom Erwachsenengewicht beträgt, sollten nach unserer Schätzgleichung Fleckviehkühe zum Zeitpunkt des Abkalbens einen Brustumfang von 210 Zentimetern aufweisen.

Das Belegungsgewicht liegt zwischen 55 und 60 Prozent vom Gewicht adulter Rinder. Wählt man im Mittel 57,5 Prozent aus, so sollte beim Fleckviehjungrind das Bedeckungsgewicht $432 \mathrm{~kg}$ betragen, was nach der empfohlenen Schätzgleichung (II) einem Brustumfang von 179 Zentimetern entspricht. Mit Hilfe dieser kann der Milchviehhalter am Tier eine Gewichtsschätzung vornehmen und unter dem Aspekt der Körperentwicklung direkt vor Ort die Entscheidung über den richtigen Zeitpunkt der Erstbesamung treffen.

ALPS, H.:

\section{Literatur}

Wiederholungsmessungen von Körpermaßen beim Rind. Schule und Berechnung Nr. 7 (1985), IV/1114

ALPS, H.; STEURER, J.:

Gewichtsschätzung mit Hilfe von Körpermaßen bei Braunvieh - Jungkühen. Allgäuer Bauernblatt 54/29 (1983), 1350-1351

BREM, G.:

Exterieurbeurteilung landwirtschaftlicher Nutztiere. Verlag Eugen Ulmer, Stuttgart, 1998

DUERST, J.U.:

Grundlagen der Rinderzucht. Julius Springer Verlag., Berlin, 1931

FLANITZER, F.:

Gewichtsermittlung weiblicher Tiere im Feld im Vergleich zur Stationsprüfung über männliche Tiere zur Zuchtwertschätzung von Vätern auf Zuwachsleistung. Hochschule für Bodenkultur Wien, Diss., 1968

GRUMMER, R. R.; HOFFMANN, P. C.; LUCK, M. L.; BERTICS, S.J.:

Effect of prepartum and postpartum dietary energy on growth and lactation of primiparous cows. J. Dairy Sci. 78 (1995), 172-180

HEINRICHS, A.J.; ROGERS, G.W.; COOPER, B.J.:

Predicting body weight and wither height in Holstein heifers using body measurements. J. Dairy Sci. 75 (1992), 3576-3581

HOFFMANN, P.C.:

Optimum body size of Holstein replacement heifers. J. Anim. Sci. 75 (1997), 836-845

HOFFMANN, P.C.; BREHM, N.M.; PRICE, S.G.; PRILL-ADAMS, A.:

Effect of accelerated postpubertal growth and early calving on lactation performance of primiparous Holstein heifers. J. Dairy Sci. 79 (1996), 2024 - 2031

LIPPERT, T.; AUMANN, J.; WILLEKE, H.: Schätzung des Gewichtes bei Kühen mit Hilfe von Körpermaßen. Tag. Ber. DGfZ u. GfT, Halle, 1994

PLATEN, M.; KROCKER, M.:

Erstkalbealter auf 24 Monate drücken - so gelingt es. Top Agrar Spezial Nr.2 (1998), 16-19

SWANSON (1960): Zitiert nach HOFFMANN, P.C. (1997)

VEAUTHIER, G.; ACHLER, B.: Färsenaufzucht: Mit 24 Monaten abkalben lassen. Top Agrar 3 (1999), R6-R9

WILLEKE, H.; GÖPPEL, H.; WILD, M.:

Gewichtige Fleckviehkühe. Rinderzucht Fleckvieh 8 (2001) Heft 3, 8

Eingegangen: 03.12.2001

Akzeptiert: 07.01.2002

Autor für Korrespondenz

Prof. Dr. HENNING WILLEKE

FHW Triesdorf

D-91746 Weidenbach-Triesdorf

E-Mail: henning.willeke@fh-weihenstephan.de 